\title{
A Presuppositional Approach to Conceptual Schemes
}

\author{
Xinli Wang \\ Philosophy Department \\ Juniata College \\ Huntingdon, PA 16652, USA \\ Email: wang@juniata.edu

\section{Ling Xu} \\ Nagoya University of Commerce and Business \\ Nagoya, Japan \\ Email: lingxu921@hotmail.com
}

\begin{abstract}
The current discussions of conceptual schemes and related topics are misguided; for they have been focused too much on the truth-conditional notions of meaning/concepts and translation/interpretation in Tarski's style. It is exactly due to such a Quinean interpretation of the notion of conceptual schemes that the very notion of conceptual schemes falls prey to Davidson's attack. I argue that what should concern us in the discussions of conceptual schemes and related issues, following the initiatives of I. Hacking, T. Kuhn, and N. Rescher, is not the truth-values of assertions, but rather the truthvalue-status of the sentences used to make the assertions. This is because the genuine conceptual innovation between alternative theories/languages does not lie in differences in determining the truth-values of their sentences, but turns on whether these sentences have truth-values when considered within the context of a competing one. The core of conceptual relativism does not consist in the claim that different conceptual schemes may yield incommensurable truth claims, but rather that different conceptual schemes may yield incompatible truth-value-status and therefore lead to distinct perceptions of reality. Conceptual schemes are no longer seen as sentential languages consisting of a set of sentences accepted as true, but rather seen as metaphysical presuppositions of presuppositional languages.
\end{abstract}

In spite of its lasting popularity among many, the notion of conceptual schemes remains murky; widespread confusion lingers over its meaning. Even so, a particular notion of conceptual schemes has been 'popularized' by Donald Davidson (1984) through his well-received criticism, namely, the Quinean linguistic model of conceptual schemes. Such a notion of conceptual schemes faces many conceptual difficulties, and thus falls prey to Davidson's attack.

Whatever the problems of the Quinean analysis might be, it is certainly not the only possible account of conceptual schemes. As many have pointed out, the Quinean model is, at best, a notion of conceptual schemes and it is not the notion to which 
many conceptual relativists commit. ${ }^{1}$ The fact that a particular analysis of conceptual schemes gets into trouble does not mean that no analysis might be satisfactory. The fall of the Quinean model does not amount to the end of the very notion of conceptual schemes as Davidson tries to convince us. This is what exactly this paper is intended to do: to offer an alternative account of conceptual schemes that is viable and does not get into the conceptual difficulties faced by the Quinean model.

\section{The Two Assumptions of the Quinean Conceptual Schemes ${ }^{2}$}

The Quinean linguistic model of conceptual schemes takes a conceptual scheme to be a set of intertranslatable sentential languages with their referential apparatus - such as predicates, quantifiers, terms - and their descriptive apparatus - mainly, sentences held to be true by the speaker. As I have argued elsewhere (Wang 2009), there are two basic assumptions behind the Quinean model that have not been widely recognized and adequately analyzed. The following is a brief recap.

First, ever since 'the linguistic turn', conceptual schemes have commonly come to be associated with languages. For Quine, a conceptual scheme is not merely associated with a language, but is rather identical with it (Quine 1981: 41). Or rather, more precisely, considering that not all languages are conceptually distinct, a conceptual scheme actually becomes, to Quine, a set of languages sharing the same conceptual makeup, whatever it may be. Furthermore, for a language to fit reality, most of its sentences have to be true. Therefore, a conceptual scheme, for Quine, eventually becomes identical to a sentential language with its sentences held to be true. Those sentential languages can be natural languages or scientific languages. ${ }^{3}$

Second, as Davidson points out, the Quinean content-scheme dualism can be reduced to a truth-value criterion of languagehood: A form of activity represents the use of a language for an interpreter if and only if it is largely true from the interpreter's perspective. Accordingly, we 'have a formula for generating distinct conceptual schemes. We get a new out of an old scheme when the speakers of a language come to accept as true an important range of sentences they previously took to be false (and, of course, vice versa)' (Davidson 1984: 188). Therefore, the difference between two schemes/languages is semantically signified by the redistribution of truth-values over their sentences. Furthermore, redistribution of truth-values over sentences between two competing languages presupposes that most sentences in the speaker's language are either true or false from the point of view of the interpreter's language no matter

To mention a few, Hacking 1982, 1983, 1992; Kuhn 1991, 1993; Rescher 1980; Lynch 1998

2 By the label 'the Quinean model of conceptual schemes,' I do not mean 'Quine's model' as such, namely, what W. V. Quine explicitly formulates and defends as a well-established theory. Rather, I intend to use the phrase to refer to one pervasive notion of conceptual schemes on the basis of the works of Quine and many other similar minded conceptual relativists such as Benjamin Whorf, David Lewis, Thomas Kuhn (before the 1980s), and John Searle. In particular, it is the notion of conceptual schemes identified, construed, and criticized by Donald Davidson.

3 A scientific language, following the convention in the discipline of philosophy of science, refers to a theoretical language employed to formulate a comprehensive scientific theory, such as Newton's physics, Aristotelian physics, Einstein's relativity theory, traditional Chinese medical theory. Feyerabend once pointed out that comprehensive scientific theories are sufficiently general, sufficiently 'deep' and have developed in sufficiently complex ways. They can be considered to some extent along the same lines as well-developed natural languages (Feyerabend 1978: 224-5). 
how disparate the two languages are. There is no truth-value gap ${ }^{4}$ between two languages.

To summarize, a Quinean conceptual scheme amounts to a sentential language construed as the totality of its sentences held to be true; the difference between two conceptual schemes consists in different distributions in truth-values such that mutual truth-preserving translation between them is doomed to failure. I contend (Wang 2009) that it is those two assumptions of the Quinean notion of conceptual schemes that play into Davidson's hand. ${ }^{5}$

\section{Emergence of a New Vision of Conceptual Schemes}

My dissatisfaction with the Quinean notion of conceptual schemes lies in a different ground from the Davidsonians. Besides many difficulties associated with construing conceptual schemes as sentential languages as I have identified elsewhere (Wang 2009), the Quinean model does not give a satisfactory account of many well-known conceptual confrontations revealed in the history of the natural sciences and cultural studies, such as Ptolemaic astronomy versus Copernican astronomy, Aristotelian physics versus Newtonian physics, Lavoisier's oxygen theory versus Priestley's phlogiston theory of combustion, traditional Chinese medical theory vs. modern Western medical theory, etc. As Haim Gaifman in his works on conceptual framework $(1975,1976$, 1984) has argued much earlier, these familiar conceptual confrontations are not confrontations between two sentential languages with different distributions of truth-values over their assertions, but rather confrontations between two competing scientific languages with different distributions of truth-value-status (whether a sentence has a classical truth-value, i.e., true-or-false) over their sentences. Consequently, the advocate of an alien conceptual scheme not only does not hold the same notion of truth as ours, but also does not agree with us on the truth-value-status of the sentences in question. The genuine conceptual innovation between alternative conceptual schemes does not lie in different distributions in truth-values, but rather in different distributions in truth-value status. The key schematic changes are those from having truth-values to having no truth-values, or vice versa. Accordingly, following Gaifman's reasoning, the core of conceptual relativism does not consist in the claim that different conceptual schemes may yield different truth claims, but rather that different conceptual schemes may yield incompatible truth-value claims and therefore lead to distinct perceptions of reality.

Dissatisfied with the Quinean model of conceptual scheme, and equally unhappy with Davidson's wholesale dismissal of the very notion of conceptual schemes, Ian Hacking, Thomas Kuhn, and Nicholas Rescher, began to explore an alternative model of conceptual schemes that, in line with Gaifman's insight, characterizes conceptual schemes in terms of truth-value-status instead of truth-values.

\subsection{Ian Hacking's Styles of Reasoning}

To his dismay, Hacking found that 'many of the recent but already classical philosophical discussions of such topics as incommensurability, indeterminacy of translation,

4 In a trivalent semantics, it is possible for a sentence to be neither true nor false, which corresponds to a lack of a classical truth-value (truth or falsity). In this case, it can be said that there is a truth-value gap regarding the sentence within a bivalent semantics. If a substantial number of core sentences of one language, when considered within the context of a competing language, lack classical truth-values, then there is a truth-value gap between the two languages. For a trivalent semantics, see Wang 1999.

5 I analyzed and criticized both the Quinean model of conceptual schemes and Davidson's attack in details in Wang 2009. 
and conceptual schemes seem to me to discuss truth, where they ought to be considering truth-or-falsity' (Hacking 1982: 49). Hacking believes that to think of conceptual schemes in terms of truth is to be on the wrong track. In fact, 'once you focus on truth rather than truth-or-falsehood, you begin a chain of considerations that call in question the very idea of a conceptual scheme' (Hacking 1982: 59).

Hacking introduces styles of scientific reasoning as an alternative to the Quinean model. A style of reasoning is characterized by introducing novelties into scientific inquiry, including new types of objects, evidence, sentences, natural laws (or, at any rate, modalities), possibilities, and new types of classification and explanation. Among all these novelties, the most notable feature that distinguishes Hacking's styles of reasoning from the Quinean notion of conceptual schemes is that each new style brings with it a new way of being a candidate for truth or falsehood. Sentences that are meaningless and cannot be used meaningfully within one style of reasoning can be asserted to be either true or false within another style (Hacking 1992: 10-17). Thus, two scientific communities committed to different styles of reasoning often find themselves experiencing a frustrating breakdown of communication when one side tries to approach to the other. For example, the medical theory of the well-known $16^{\text {th }}$ century Swiss alchemist and physician Paracelsus, which exemplifies a host of hermetic interests within the Northern European Renaissance tradition, makes little sense to modern Westerners. To us, a Paracelsian sentence, 'syphilis should be treated by a salve of mercury and by internal administration of the metal, because the metal mercury is the sign of the planet, Mercury, and that in turn signs the market place, and syphilis is contracted in the market place,' and other numerous Paracelsian sentences like this are not among our candidates for truth-or-falsehood. We cannot even claim that Paracelsus wrote falsely since we simply cannot assign any truth-value to a great many of his sentences.

In contrast with the Quinean notion of conceptual schemes, Hacking's styles of reasoning have a few distinctive features. (a) Unlike the Quinean conceptual schemes that assign truth-values to sentences, the styles of reasoning assign truth-value-status to sentences or determine 'what is taken to be a legitimate candidate for truth or falsity'. (b) The styles of reasoning are not 'sets of sentences held to be true', but 'sets of sentences that are candidates for truth or falsehood' (Hacking 1982: 58, 64). (c) Accordingly, the styles of reasoning are not sets of beliefs or propositions about the world, but rather the way beliefs or propositions are proposed and defended. (d) The Quinean scheme is often characterized as a language confronting reality as in the fitting model while 'a style is not a scheme that confronts reality' (Hacking 1982: 64). Therefore, such a notion would not fall into the third dogma of scheme-reality that Davidson attacks.

If Hacking were forced to use the term 'conceptual schemes', he would define a conceptual scheme as 'a network of possibilities, whose linguistic formulation is a class of sentences up for grabs as true or false' (Hacking 1983: 71). Consequently, for 
Hacking, two schemes are distinct when the core sentences ${ }^{6}$ of one scheme are not held to be true or false in another scheme, while for a Quinean, 'two schemes differ when some substantial number of core sentences of one scheme are not held to be true in another scheme' (Hacking 1982: 58).

\subsection{Thomas Kuhn's Lexical Structure}

Following Hacking's initiative, Kuhn realizes the importance of the distinction between the notion of truth-value and the notion of truth-value-status in his latest interpretation of incommensurability. In 1991, Kuhn wrote,

Since that time (referring to the publication of Hacking's paper on relativism in 1982 - author), I have been gradually realizing (the reformulation is still in process) that some of my central points are far better made without speaking of statements as themselves being true or as being false. Instead, the evaluation of a putatively scientific statement should be conceived as comprising two seldom-separated parts. First, determine the status of the statement: is it a candidate for true/false? To that question, as you'll shortly see, the answer is lexicon-dependent. Second, supposing a positive answer to the first, is the statement rationally assertable? To that question, given a lexicon, the answer is properly found by something like the normal rules of evidence (Kuhn 1991: 9).

These two stages of theory evaluation roughly correspond to the two phases of scientific development as defined by Kuhn's early works (before 1980s), namely, revolutionary science and normal science. Now Kuhn realizes that the landmark of a scientific revolution (paradigm shift) is not the redistribution of truth-values, but reassignment of truth-value-status. For instance, when a Newtonian finds Aristotelian sentences hard to understand, the trouble is not that he thinks Aristotle wrote falsely, but that he cannot attach truth or falsity to a great many of the Aristotelian core sentences. Consequently, a truth-value gap occurs between the Newtonian language and the Aristotelian language. Similar occurrences of truth-value gaps abound in the history of science. 'Though the originals were candidates for true/false, the historian's later restatements - made by a bilingual speaking the language of one culture to the members of another-are not' (Kuhn 1991: 9).

Kuhn substitutes lexicon (lexical taxonomy, taxonomic structure) for the Quinean notion of conceptual schemes. The lexicon of a scientific theory is, briefly, a mental 'module in which each member of a speech community stores the kind-terms and kind-concepts used by community members to describe and analyze the natural and social worlds" (Kuhn 1993: 325). For Kuhn, "the "very notion" of a conceptual scheme is not that of a set of beliefs but of a particular operating mode of a mental module prerequisite to having beliefs, a mode that at once supplies and bounds the set of beliefs it is possible to conceive' (Kuhn 1991: 5). Like Hacking's styles of reasoning, the lexicon of a scientific language determines the truth-value-status of its sentences.

Each lexicon makes possible a corresponding form of life within which the truth or falsity of propositions may be both claimed and rationally justified, ... With the Aristotelian lexicon in place, it does make sense to speak of the truth

6 As it will become clear later that the core sentences of a scientific language are ones that directly presuppose one or two metaphysical presuppositions of the language, such as Hacking's style of reasoning. Those sentences would lack truth-value when viewed within the context of a competing language. 
or falsity of Aristotelian assertions in which terms like 'force' or 'void' play an essential role, but the truth values arrived at need have no bearing on the truth or falsity of apparently similar assertions made within the Newtonian lexicon (Kuhn 1993: 330-31).

\subsection{Nicholas Rescher's Factual Commitments}

Unsatisfied with the Quinean notion of conceptual schemes and Davidson's argument from translatability against the notion, Rescher proposes a new version of conceptual schemes within 'a three-valued framework of truth-values, one that adds the neutral truth-value $(I)$ of interdeterminacy or indefiniteness to the classical values of truth and falsity ( $T$ and $F)^{\prime}$ ' (Rescher 1980: 332).

As the term suggests, a conceptual scheme is the mode of operation of concepts. For Rescher, the notion of concepts does not refer to mysterious Platonic entities, nor to Kantian a priori mental schemes. Instead, 'concept' is a generic term used to denote all that is conceptual, such as 'categorical frameworks' (descriptive and explanatory mechanisms), 'taxonomic and explanatory mechanisms', 'fundamental concepts', and 'modes of classification, description, explanation'. Just as Quine has convinced us that there is no clear distinction between the analytical/conceptual and the synthetic/empirical, our concepts are always correlative with and embedded in a substantive view of how things work in the world. In other words, concepts are themselves loaded with substantial empirical/factual commitments. Especially our conceptual schemes for operation in the factual domain (in science) come to be correlative with a set of factual commitments or presuppositions, which constitute the essence of a conceptual scheme. In this sense, the factual commitments embedded within a scientific theory/language are its conceptual scheme. 'Schemes differ in just this regard-in undertaking different sort of factual commitments', or in a different way of conceptualizing the purported facts (Rescher 1980: 329-31).

Like Hacking's styles of reasoning and Kuhn's lexicons, factual commitments determine whether sentences have truth-values. Since alternative conceptual schemes come with (or are) different factual commitments underlying the languages, one sentence that has a truth-value within one scheme and therefore can be used to make an assertion about the world may have no truth-value at all within a competing scheme and therefore could not be used to make an assertion.

Consequently, Rescher reaches the same conclusion as Hacking and Kuhn about the scheme differentiation. 'The issue of scheme innovation at bottom turns out not on differences in determinate truth-values but on the having of no truth-value at all'. More precisely, 'the key schematic changes are those from a definite (classical) truth-status to $I$ (i.e., from $T$ or $F$ to $I$ ) or those in the reverse direction (i.e., from $I$ to $T$ or $F$ )' (Rescher 1980: 332). This is why the Quinean notion of conceptual schemes cannot reveal the genuine conceptual innovation.

If the conceptual scheme $\mathrm{C}^{\prime}$ is to be thought of as an alternative to $\mathrm{C}$ along the lines we have in view, then one cannot think of $C^{\prime}$ as involving a different assignment of truth-values to the (key) propositions of C. One must avoid any temptation to view different conceptual schemes as distributing truth-values differently across the same propositions. The fact-ladenness of our concepts precludes this and prevents us from taking the difference of schemes to lie in a disagreement as to the truth-falsity classification of one selfsame body of these or doctrines (Rescher 1980: 331). 
For this reason, Rescher contends that the key contrast between competing conceptual schemes is not between affirmation (to be true) and counter-affirmation (to be false), but rather between saying something (to be either true or false) and saying nothing (to be neither true nor false). As such, two schemes do not dispute over the same thing or fact; rather, they are about wholly different kinds of things and facts. If so, Davidson's charge about the third dogma is off target since there is no common content for two conceptual schemes to process.

\section{Presuppositional Languages}

I believe that Ian Hacking, Thomas Kuhn, and Nicholas Rescher present a very promising model of conceptual schemes different from the Quinean model. However, they have only offered scattered insights. Many details need to be worked out in order to make it a full case. This is why these insights have not yet gained their deserved attention. It is my intention to give them a coherent formulation by providing a coherent semantic framework. I hope this treatment will help this new version of conceptual schemes gain the acceptance that it deserves.

If the primary function of the proposed conceptual schemes-Hacking's styles of reasoning, Kuhn's lexicons, or Rescher's factual commitments - is to determine the truth-value-status of the sentences involved, then we need a workable semantic truth-value condition. However, Hacking, Kuhn, and Rescher fail to provide one. For example, Hacking fails to tell us how the truth-value-status is style-of-reasoning dependent. How can the introduction of new types of objects, evidences, classifications, and explanations change the way in which sentences are candidates for truth or falsehood? Fortunately, there is a semantic theory that is useful in constructing a badly needed truth-value condition and explaining the occurrence of truth-value gaps between competing conceptual schemes, namely, R. G. Collingwood's (1940, ch. 4) and P. F. Strawson's (1996) theory of semantic presuppositions. Although the notion of semantic presuppositions will play an essential role in my presuppositional approach to conceptual schemes, it is still a fiercely contested notion in post-Strawsonian literature. However, a full clarification and defense of the notion in detail would move us far beyond the scope of the current paper, which I have done in an earlier paper (The reader who is interested in a full overview of the notion can refer to Wang 1999). Here I can only introduce the notion briefly below.

As Collingwood observed, every statement is made potentially in answer to a question; and every question involves a presupposition. Thus, every statement involves a presupposition. For example, sentence $K$ :

$(K)$ 'The present king of France is bald',

could be used to answer a potential question:

$\left(Q_{k}\right)$ 'Does the present King of France have hair?'

The question in turn presupposes sentence $K_{a}$ :

$\left(K_{a}\right) \quad$ 'The present king of France exists'.

Of course, a statement may have many different presuppositions. For instance, $K$ has at least four different presuppositions, i.e., $K_{a}$,

$\left(K_{b}\right)$ 'There is a country called France';

$\left(K_{c}\right)$ 'A person can have hair'; and 
$\left(K_{d}\right)$ 'A subject (such as 'a person') can have a property (such as 'of having hair')'.

What happens if a presupposition of a statement does not hold? For Collingwood, if a presupposition of a question is not made, then the question simply does not arise. That implies that we cannot judge the truth-value of the corresponding initial statement that the question addresses since it is not even a proposition. Strawson draws the same conclusion. Based on Strawson's trivalent semantics (1996), a semantic presupposition of a sentence has to be held true in order for the sentence to be true or false. In other words, the truth of a presupposition of a sentence is necessary for the truth or falsity of the sentence. For example, $K$ is true or false only when $K_{a}$ is true; otherwise, $K$ is neither true nor false (has no classical truth-value).

More significantly for our later discussion is Collingwood's distinction between relative and absolute presuppositions. A relative presupposition is a proposition that could be questioned or verified within a certain domain of inquiry or a language community, such as $K_{a}, K_{b}, K_{c}$ for our common-sense non-metaphysical way of thinking. In contrast, some fundamental presuppositions are taken for granted by a language community that cannot be verified or falsified within that community; these are absolute presuppositions for the language community. For instance, $K_{d}$ is an absolute presupposition to be taken for granted in all of our non-metaphysical inquiry. Such absolute presuppositions are abundant in science. It is not a new idea to argue that a scientific language is loaded with absolute presuppositions, such as the existence of phlogiston within the language of phlogiston theory, or the existence of absolute space and time for Newtonian physics. In general, we can identify at least three primary types of absolute presuppositions underneath the core sentences of many paradigmatic scientific languages as follows:

A. Existential Presuppositions: Many core sentences of a scientific language presuppose the existence of some theoretical entities postulated by the corresponding theory. For example, the language of phlogiston theory presupposes the existence of phlogiston. To say, 'The element a is not richer in phlogiston than the element b' presupposes that 'Phlogiston exists'. Similarly, the existence of the yin and the yang, as well as the five elements, underlies many core sentences of the language of traditional Chinese medical theory. I will call this kind of presuppositions of a scientific language, which are about the theoretical entities that a language community believes to exist in the world, existential presuppositions.

B. State-of-Affairs Presuppositions (and the Modes of Reasoning): Some fundamental assumptions underlie a scientific language about the existential state of the world perceived by the language community, such as uncertainty relations in the quantum theory or the postulate of absolute space and time and the second law of motion in the language of Classical Newtonian physics. Those are the fundamental shared state-of-affairs presuppositions of a scientific language.

More significantly, we can often find a unique mode of reasoning associated with some hidden state-of-affairs presuppositions of a scientific language. Recall that Ian Hacking has identified different styles of scientific reasoning within the Western scientific tradition, such as the Euclidean style of thought in ancient Greece, and the Galilean style of reasoning in the modern time (Hacking 1982: 48-9). Left unresolved by Hacking, however, is how a form of reasoning, no matter what it is, can determine the truth-value status of sentences. We know that, according to Strawsonian notion of se- 
mantic presupposition, the truth-value status of a sentence is determined by the truth-value of its presupposition(s). A presupposition of a sentence is an assumption about the existential state of the world. This suggests that as far as its function of truth-value-status determination is concerned, a style of reasoning has to be associated with some kind of assumptions. Therefore, it is reasonable to hypothesize that for any specific style of reasoning within a cultural / intellectual tradition, there are some associated hidden state-of-affairs presuppositions. More precisely, it is imaginable that every style of reasoning is embedded in a cosmology constituted by some (hidden or explicit) state-of-affairs presuppositions. During the historical development of a tradition, these initially explicit state-of-affairs presuppositions operate constantly and eventually become treated as constants within it to the extent that they become incorporated into the conception of understanding, and consequently generate a unique way of understanding the world. A unique style of reasoning eventually evolves from the same process. Then to say that a style of reasoning determines the truth-value status of sentences is actually to say that the associated hidden state-of-affairs presuppositions determine it. In this way, the internal conflict between two merits of a style of reasoning - i.e., as a form of reasoning and as a truth-value status determinator - can be resolved. To distinguish this modification of the notion of the style of reasoning from Hacking's, the former is herein referred to as 'the mode of reasoning'.

C. Categorical Presuppositions: A categorical presupposition is a specific category system that describes the structure of the world as perceived by a language community. One kind of category system deserves our special attention, namely, Kuhn's lexical taxonomy. Different scientific languages often presuppose different lexical taxonomies, such as the Copernican taxonomy (in which planets include the earth but not the moon and the sun) versus the Ptolemaic taxonomy (in which the moon and the sun were in the extension of the kind 'planets' but the earth was not). The lexical taxonomy of a scientific language actually functions as a system of shared fundamental sortal presuppositions ${ }^{7}$ of the language that set the boundaries for the categories of its core predicates. Take the Ptolemaic sentence $L$ : 'All the planets revolve about the earth' as an example. $L$ presupposes at least one assertion about the categorical status of the earth. That is, $L_{a}$ : 'The earth is not a planet', which is analytically implied by the verb 'revolve' in $L . L_{a}$ is one fundamental sortal presupposition of the Ptolemaic language about the category of the predicate, '__ is a planet', which excludes 'the earth' from the category of "planets'. 8

I call a theoretical language, such as a scientific language, loaded with those absolute presuppositions a presuppositional language ('P-language' in brief hereafter). Even natural languages are p-languages to some extent. That the sun exists, that it rises

7 Besides logical presuppositions, a sentence may presuppose many different sortal presuppositions. For example, $S$ : 'My soul is red' presupposes $S 1$ : 'A soul is capable of being colored'. $S 1$ in turn presupposes $S 2$ : 'Some non-sense-perceivable entities are capable of being colored'. If a sortal presupposition is so fundamental to a language that it sets the boundary for the category of its predicates, then it is $a$ fundamental sortal presupposition for the language. To know more on the notion of sortal presuppositions, see Martin 1975.

8 Based on Kuhn's contextual theory of meaning, the meaning of 'planet' is determined by the content and taxonomy of Ptolemaic theory. One might wonder if the Ptolemaic 'planet' means differently from 'planet' used by Copernican theory, then the Ptolemaic sentence L and the Copernican sentence, 'It is not true that all the planets revolve about the earth', are not logically incompatible. If so, how can the two theories be incommensurable as what the author clearly has in mind. Our critic here confuses incommensurability with incompatibility. For the distinction, please refer to Wang 1998. 
and sets periodically, may in many everyday discourses count as an inevitable presupposition. Denial of them would play chaos with everyday communicative activity.

As an illustration of a typical p-language, consider the language of traditional Chinese medical theory (hereafter CMT). During its more than two-thousand-year development, CMT has established a complete conceptual system including its own physiological theory, pathological theory, diagnosis, and treatments. Its physiological / pathological basis consists of the yin-yang doctrine, the five-element doctrine, the viscera doctrine, and the jingluo doctrine. According to the yin-yang doctrine, it is the balance between the yin and yang parts of the human body that ensures its normal function and health. Loss of the yin-yang balance leads to diseases. Treatment is therefore a matter of restoring the balance between the yin and the yang. For example, CMT holds that the spleen is responsible for transport and conversion. The spleen effects the upward movement of vital substances and controls blood. Many spleen diseases are caused by the imbalance between the yin and the yang within the spleen, which is manifested as either an asthenic or a sthenic spleen. The cure for these diseases lies in the nourishment of the spleen to restore the yin-yang balance.

The three types of absolute presuppositions are identifiable within CMT. First, the existence of the yin and the yang as well as the five elements is an existential presupposition of CMT. Both underlie numerous core sentences of the language of CMT. Second, CMT has its own unique medical category system. For example, all symptoms related to diseases are classified as eight principal syndromes, which can be grouped further into four matched pairs: the yin versus the yang syndrome; the superficial versus the interior syndrome; the cold versus the heat syndrome; and the asthenia versus the sthenia syndrome. The yin syndrome governs the superficial, the asthenia, and the cold syndrome, while the yang syndrome controls the interior, the sthenia, and the heat syndrome. Therefore, all diseases arising from loss of the yin-yang balance can be diagnosed as the result of either a yin-syndrome or yang-syndrome.

Third and most significantly, CMT embodies the premodern Chinese mode of reasoning. It evolved from the yin-yang cosmology, which consists of at least three doctrines. One is the yin-yang doctrine: all things and events in the universe are produced and controlled by two forces or principles, namely, the yin and the yang. The yin, which represents the negative, passive, weak, and destructive side of the universe, is associated with soft, coldness, cloudiness, rain, winter, femaleness, and what is inside and dark. The yang, which represents the positive, active, strong, and constructive side of the universe, is associated with hardness, heat, sunshine, spring and summer, maleness, and what is outside and bright. Another doctrine of CMT is the five-element doctrine: everything is made of five elements, Metal, Wood, Water, Fire, and Earth. Things succeed one another as the five agents take their turns. The third doctrine of CMT is the principle of preestablished harmony: the universe is treated as an organic whole composed of ten related parts - the Tian, the Earth, the yin, the yang, wood, fire, soil, metal, water, and human beings. Within this organic structure, everything undergoes constant transformation. The final cause of these transformations is the yin and the yang. The yin and the yang are of opposing nature. If one flourishes, then the other declines. In addition, not only are things related generally, but also they activate each other. This mutual correlation among things presupposes that all forces and things in the universe are harmonized. This preestablished harmony manifests itself best in certain correlations in the yin or yang operations of nature with events in human affairs. 
During the development of Chinese civilization, these beliefs in the yin-yang cosmology had operated constantly in the premodern Chinese intellectual tradition and eventually were internalized into the premodern Chinese way of thinking, which Sinologists call 'associated thinking'. This kind of thinking can be best characterized as a specific way of understanding the world within the premodern Chinese tradition. According to this way of thinking, in brief, the world is composed of and is operated by the yin-yang and the five elements. Human beings, holding the most exalted position in it, become attuned to the world to such an extent that they become a shadow of the universe, and are unified with it. Because of such a close relationship between human beings and the universe, ancient Chinese did not treat their surrounding world as being composed of external objects that you can love, rebel against, or control. They appreciated the blessings of Tian and Earth (associated with the yang part of the universe) and accepted the punishment (associated with the yin part of the universe) that befell them. Consequently, for ancient Chinese, understanding the universe was intertwined with achieving attunement with it. Both are related so closely that attunement had actually become a necessary condition of understanding.

During the historical development of this kind of associated thinking, the link between attunement and understanding was constantly incorporated into the mainstream values of ancient Chinese culture. Consequently, it eventually became institutionalized as a dominant mode of reasoning. It not only determined what facts count as evidence for justification, but also determined what states of affairs count as accepted facts. For example, according to this mode of reasoning, a specific interaction between the yin-yang parts in the human body and the yin-yang forces in Tian counts as a possible fact. Many symptoms can be attributed to the associations between natural forces and changes - which represent the yin or the yang principles of the universe - and the yin-yang parts of the human body that are supposed to correspond to the former. For instance, according to Han Confucians, when Tian is about to make rain (representing the yin) fall, people feel sleepy. This is because when the yin force in Tian and Earth begins to dominate, the yin in the human body takes over. The association between the yin and rain causes increased sleepiness among people.

\section{Schemes of Metaphysical Presuppositions}

As I have argued above, the conceptual core of a P-language consists of a set of absolute presuppositions underlying the core sentences of the language, which I call metaphysical presuppositions of the language ('M-presuppositions' hereafter). The scheme of M-presuppositions of a P-language is what I will call the conceptual scheme of the language. A conceptual scheme is, briefly put, a conceptual framework that schematizes experience in terms of its metaphysical presuppositions of existents, states-of-affairs, modes of reasoning, and categorization to form a theory.

The M-presuppositions of a P-language are contingent factual presuppositions about the world perceived by a language community. Since they are absolute presuppositions within a certain school of thought, the ordinary scientist's business during the normal science phase is not to question or verify them, but only to presuppose them. To presuppose them is more than just to accept them as true since one can accept the truth of one assumption after deliberation or verification. To presuppose them here means to take their truth for granted without questioning. As Collingwood points out, the logical efficacy of an absolute presupposition 'does not depend on its being verifiable, because it does not depend on its being true: it depends only on its being supposed' 
(1940: 32). I would say that the M-presuppositions of a P-language are universally true when perceived within the language in the sense that denial of them signifies a complete breakdown of informative use of the language.

The essence of a P-language consists in its scheme of M-presuppositions. It is the M-presuppositions of a P-language that determine whether its sentences used to describe states of affairs have truth-values. To say that a sentence of a P-language has a truth-value is to say that the state of affairs designated by the sentence is a possible fact. To ask about possible facts is to ask whether a language is fit to describe the world perceived through it. Therefore, it is the M-presuppositions of a P-language, in terms of determining the truth-value status of its core sentences, that determine whether the language is fit to describe the world perceived by the language community. In this sense, these M-presuppositions are actually the ontological commitments of the language. The essential job of a P-language is to form a conceptual setup specified by its M-presuppositions with which to describe the world under consideration. To adopt a P-language is to presuppose one specific cosmology.

To treat a conceptual scheme as a scheme of M-presuppositions of a P-language is advantageous for several reasons. First, by doing so we can catch the essence of scheme-cont significantly, although Ian Hacking, Thomas Kuhn and Nicholas Rescher realize correctly that scheme change and differentiation do not consist in redistribution of trent dualism; that is, conceptual schemes are 'necessary for the constitution of experience, not just necessary to control and predict experience' (Rorty 1982: 5). A conceptual scheme is not what we experience, what we believe consciously, but what makes our experience and beliefs possible since some conceptual structures are logically presupposed by all experiences and beliefs of a language community.

Second, a conceptual scheme is closely associated with a language, but not identical with a sentential language as the Quinean model claims. As P. F. Strawson has argued, a presupposition of a sentence is not a part of the sentence, and is not even logically entailed by the sentence. By the same token, an M-presupposition (i.e., 'There exists phlogiston') of a sentence (i.e., 'The element $a$ is not richer in phlogiston than the element $b^{\prime}$ ) is not a part of the sentence. Thus, strictly speaking, the M-presuppositions of a P-language (i.e., the language of phlogiston theory), which are important components of the corresponding scientific theory (phlogiston theory), are not parts of linguistic setup of the P-language; although they are, as its ontological commitments, surely parts of its deep metaphysical setup. Therefore, our conceptual scheme is not a sentential language, but the conceptual core of a P-language.

Third, 'a conceptual scheme' is a broader notion than 'a set of concepts' if 'concept' is construed either in a normal sense - namely, an abstract entity, the meaning, the interpretation, or the disposition associated with a term ('causation', 'a tree', 'a game') _ or in a functional sense - namely, whatever composes the propositional content of our assertions and beliefs. Instead, 'a conceptual scheme' means 'a scheme of what is conceptual', which includes basic concepts, categorical frameworks (lexical structures or taxonomies), modes of reasoning and justification, ways of thinking, description, and explanation, and some fundamental factual commitments (universal principles, existential presumptions). Those are M-presuppositions of a P-language.

Last but mostuth-values as the Quinean model tells us, but are semantically correlated with the redistribution of truth-value-status, they fall short of specifying a truth-value condition to sufficiently explain the change of the truth-value-status. If a conceptual scheme is construed as a scheme of M-presuppositions, it is not hard to understand why the core sentences of a P-language $\mathrm{PL}_{1}$ could be truthvalueless when 
viewed from the perspective of a competing P-language $\mathrm{PL}_{2}$ that suspends the M-presuppositions of $\mathrm{PL}_{1}$. I will construct such a truth-value condition based on Strawson's theory of semantic presuppositions in section 7 .

\section{A semantic Correlate of Alternative Conceptual Schemes}

One question remains regarding the individuation of a scheme of M-presuppositions: How can we distinguish one scheme of M-presuppositions from another? What sorts of changes in a scheme of M-presuppositions would constitute an alternative scheme? When do we know that we encounter an alternative scheme of M-presuppositions? It is time to address those concerns.

As we have argued in section 3, scheme innovations do not consist in difference in truth-values as the Quinean model assumes, but in change of truth-value status. As an illustration, let us consider two competing medical languages, i.e., contemporary Western medical language (WML) and traditional Chinese medical language (CML). Evolving within two substantially different cultures, intellectual traditions, and linguistic settings, these two medical communities experience their surrounding worlds differently, and describe and explain their own experience in terms of disparate conceptual mechanisms - different medical concepts, disjointed medical categorical systems, distinct modes of reasoning and rational justification, and different ways of conceptualizing facts (which count as presumptive facts). Consequently, these disparate conceptual mechanisms are bound up with different worldviews in such a way as to make intellectual contact with one another difficult.

We must avoid any temptation to view these two different conceptual schemes as distributing truth-values differently across the same propositions. To illustrate, imagine that a Chinese physician Dr. Wong, when asked why people tend to become sleepy on rainy days, claims that

$\left(C_{a}\right)$ the association of the yin and rain makes people sleepy.

What is a likely response of a typical practitioner of Western medicine, say, Dr. Smith? To see his possible responses, we need to consider two different hypothetical situations.

First, suppose that Dr. Smith knew nothing about CML and had no background in Chinese culture, philosophy, and language. Under these suppositions, he would certainly not say, 'No, what you have said is simply false'. He could not understand $C_{a}$ since he could not grasp the way in which the assertion was proposed and justified. It was, hence, very likely that he would say something like, 'I do not know what you are talking about'.

Second, assume that Dr. Smith happened to know CML and the yin-yang doctrine in ancient Chinese philosophy, and was able to comprehend the pre-modern Chinese mode of reasoning. Suppose further that he did not adopt the yin-yang doctrine and the corresponding mode of reasoning. If we asked him whether he thought Dr. Wong's assertion is true or false, would he directly deny the assertion by replying, 'No, $C_{a}$ is false'? I do not think so for the following considerations. We can reformulate Dr. Smith's answer as follows:

$\left(C_{b}\right)$ It is not the association of the yin and rain that makes people sleepy. ${ }^{9}$

Then, both $C_{a}$ and $C_{b}$ presuppose a third sentence,

9 It is debatable whether a negative sentence like $C_{b}$ presupposes $C_{c}$. For a clarification and defense of the current treatment of negative sentences, please refer to Wang 1999. 
$\left(C_{c}\right)$ There is a fundamental element, force, or principle in the universe, namely, the yin, and there is a pre-established connection between the human body and natural forces.

Dr. Smith cannot simply deny $C_{a}$ on the spot, for such a denial seems to set a trap for him. He will hesitate to deny $C_{a}$ directly. Dr. Smith's uneasiness about how to answer $C_{a}$ could have been strengthened if he had realized the difference between the truth-value-status of $C_{a}$ and a syntactically similar sentence $U$ :

$(U)$ The association of the falling of the American stock market and rain makes people sleepy.

It is intuitively recognizable that $U$ is untrue in a way different from the non-truth of $C_{a}$. The non-truth of $C_{a}$ is due to the failure of its 'felt implication', namely, $C_{c}$. But the non-truth of $U$ is caused by a false connection between unrelated events. Therefore, it is possible for the negation of $U$ (It is false that the association of the falling stock market makes people sleepy) to be true while the negation of $C_{a}$, namely, $C_{b}$, to be untrue. In addition, due to his uneasiness about how to answer $C_{b}$, Dr. Smith would very likely respond to the question by saying, 'I do not think I can answer the question since there is no such thing as the yin'. To assert that there is no such thing as the yin is certainly not to contradict $C_{a}$. He is rather giving a reason for saying that question $Q_{c}$ does not arise. ${ }^{10}$

In both situations, we reach the same conclusion: for our Western physician, the question of whether $C_{a}$ is true or false simply does not arise. A similar analysis can be extended to other core sentences of CML. We can apply Nicholas Rescher's observation of scheme-differentiation in general (Rescher 1980) to the current case: the confrontation between the two medical languages does not lie in the sphere of disagreement or conflict of the sort arising when one language holds something to be true that the other holds to be false. ${ }^{11}$ The difference between them is not that WML has a different theory of the operation of the yin and the yang from that of its Chinese counterpart, or that the Chinese physician says different things about bacteria and viruses. It is not that they say the same thing differently, but rather that they say different things. To the Western physician, many core sentences of CML simply have no truth-values. Consequently, there is a truth-value gap between the two languages. Such a truth-value gap between the two medical languages indicates semantically that we encounter two alternative medical conceptual schemes. Similar observations apply to other competing conceptual schemes.

So far, we have identified a semantic correlate of two alternative conceptual schemes: the occurrence of a truth-value gap between two P-languages embedded with the schemes. Suppose that $\left\langle\mathrm{MP}_{1}>\right.$ and $<\mathrm{MP}_{2}>$ are two distinct schemes of M-presuppositions of two disparate $\mathrm{P}$-languages $\mathrm{PL}_{1}$ and $\mathrm{PL}_{2}$. We can define a pair of compatible schemes of M-presuppositions as:

10 The above analysis is essentially a rehearsal of Strawson 1996. For a defense of such a Strawsonian analysis of presupposing sentences like $C_{a}, C_{b}$, and $C_{c}$, please refer to author's Wang 1999.

11 One may wonder that the confrontation between the two medical languages is a matter of truth and falsity; is it that the truth and falsity of the M-presuppositions of the two P-languages are at stake? Remember I have pointed out that the M-presuppositions of a P-language are not parts of linguistic setup of the P-language; although they are, as its ontological commitments, surely parts of its deep metaphysical setup. This is why I believe that the confrontation between the two P-languages does not lie in the sphere of disagreement or conflict in truth-values. 
$<\mathrm{MP}_{1}>$ and $<\mathrm{MP}_{2}>$ are compatible iff $<\mathrm{MP}_{1}>$ and $<\mathrm{MP}_{2}>$ are or could both be held to be true by the advocates of $\mathrm{PL}_{1}$ and $\mathrm{PL}_{2}$.

Consequently, the members of two language communities with compatible schemes of M-presuppositions agree on the truth-value status of sentences of the alternative language, although they may differ in assigning truth-values to the same sentences. In contrast, two schemes of M-presuppositions of two different P-languages are incompatible if the M-presuppositions of one language are categorically rejected by the advocate of the other. Two incompatible schemes of M-presuppositions assign opposite truth-value status to numerous core sentences of one language under consideration. Accordingly, two P-languages $\mathrm{PL}_{1}$ and $\mathrm{PL}_{2}$ are compatible if their $\mathrm{M}$-presuppositions $<\mathrm{MP}_{1}>$ and $<\mathrm{MP}_{2}>$ are compatible with one another; otherwise the two languages are incompatible.

\section{M-Presuppositions and Truth-Value Conditions}

A further question is, 'What causes a truth-value gap between two competing P-languages?' To answer this question, it is useful to distinguish truth-value conditions from truth-conditions. The usual theories of truth, such as the correspondence theory, will not work here since they are semantic theories about truth conditions and can only be used to determine the truth-value of a statement. ${ }^{12}$ According to Tarski's semantic theory of truth, a theory of truth for a language L is a set of axioms that entails, for any sentence $S$ in L, a statement of conditions under which it is true. If there is a definition of the truth predicate " is true in L' satisfying Tarski's Convention T,

(Con-T) $s$ is true in L iff $p$,

then there is a theory of truth for L. When ' $s$ ' is replaced by a canonical description of a sentence $S$ in an object language L and ' $p$ ' by a sentence $P$ of a metalanguage $\mathrm{M}$, the corresponding T-sentence, ' $S$ is true in L iff $P$ ', gives us $S$ 's truth conditions in L.

However, what is at issue here is not whether a statement is true, but rather whether a given string of words is assertable (hence qualifies as a statement), or whether a sentence has a truth-value. What we need is not an account of truth conditions, but an account of truth-value conditions. As I see it, Collingwood's and Strawson's notion of semantic presupposition provides us with a basic theoretical framework for such an account. Recall that a sentence may have many different presuppositions. The conjunction of all the presuppositions of a sentence, if true, is sufficient for the truth or falsity of the sentence. For instance, the conjunction of $K_{a}, K_{b}, K_{c}$, and $K_{d}$, if true, is sufficient for $K$ to be true or false. But if any one of them is not true, then $K$ is neither true nor false. The above reasoning can be formalized as follows: Suppose that $P_{\mathrm{i}}(\mathrm{i}=$ $1,2, \ldots, n)$, when considered within a P-language PL, is a complete list of all the presuppositions of a sentence $S$ of PL. Then $S$ 's sufficient presupposition is, $\mathrm{P}=\left(\mathrm{P}_{1} \& \mathrm{P}_{2}\right.$ $\left.\ldots \& \mathrm{P}_{\mathrm{n}}\right)$. It can be symbolized as: $\vDash \mathrm{PL}_{\mathrm{PL}}\left[\left(\mathrm{T}_{\mathrm{PL}}(\mathrm{S}) \vee \mathrm{F}_{\mathrm{PL}}(\mathrm{S})\right) \mathrm{T}_{\mathrm{PL}}\left(\mathrm{P}_{1} \& \mathrm{P}_{2} \ldots \& \mathrm{P}_{\mathrm{n}}\right)\right] .{ }^{13}$ If any one of the presuppositions $P_{i}(\mathrm{i}=1,2, \ldots, \mathrm{n})$ is untrue, then $S$ will be neither true nor false. If all the presuppositions are true, then $S$ is true or false. Unlike Tarski's Convention $\mathrm{T}$ used to determine the truth-value of a sentence in a language, the above

12 Here we are only concerned with the epistemic dimension of truth (about truth conditions), not about the semantic dimension of truth (about the metaphysical nature of truth).

$13 \mathrm{~T}_{\mathrm{PL}}(\ldots)$ ) refers to the truth predicate ' $\_$is true in PL'. Similarly, $\mathrm{F}_{\mathrm{PL}}(\ldots)$ refers to the falsity predicate. 
formula (dubbed 'Convention P') can be used to determine the truth-value-status ${ }^{14}$ of a sentence in a language. To express this in a format analogous to Convention $\mathrm{T}$,

(Con-P) A sentence $S$ is true or false when considered within a language PL if and only if ' $S$ 's sufficient presupposition $P$ is true in PL: $F$ PL $\left[\left(\mathrm{T}_{\mathrm{PL}}(\mathrm{S}) \vee\right.\right.$ $\left.\left.\mathrm{F}_{\mathrm{PL}}(\mathrm{S})\right) \leftrightarrow \mathrm{T}_{\mathrm{PL}}(\mathrm{P})\right]$.

To say that a sentence is true (or false) in terms of some given truth conditions presupposes that it has a truth-value in terms of some given truth-value conditions.

According to Convention P, whether a sentence $S$ has a truth-value, when considered within the context of P-language PL, depends on the truth-value of $S$ 's sufficient presupposition in PL. This means that the truth-value-status of a sentence is relative to the specific P-language within which the sentence is considered. It is the P-language that creates the possibility of truth or falsity. More precisely, since the M-presuppositions of a P-language are its absolute presuppositions, which are self-evident and unquestionable for its practitioners, the truth-value-status of the core sentences of the language is determined by its $\mathrm{M}$-presuppositions. If the speakers of $\mathrm{P}$-language $\mathrm{PL}_{1}$ are unable to recognize and comprehend, or are able to comprehend but do not accept the M-presuppositions of an alien P-language $\mathrm{PL}_{2}$, then the core sentences of $\mathrm{PL}_{2}$, when considered within the context of $\mathrm{PL}_{1}$, will lack truth-values. Consequently a truth-value gap occurs between $\mathrm{PL}_{1}$ and $\mathrm{PL}_{2}$.

\section{Responses to Davidson}

One might wonder how our presuppositional model of conceptual schemes fares against Davidson's attack. Clearly, the model effectively sidetracks Davidson's verificationist arguments by removing the two basic assumptions of the Quinean model. According to our presuppositional model, a conceptual scheme is not identical with a sentential language, but rather with a scheme of M-presuppositions of a P-language. Thus, sentential-language translatability can no longer be used as a criterion for the identity of conceptual schemes. In addition, an alien conceptual scheme is not a set of sentences taken to be 'largely true' or to 'fit' reality. Consequently, content-scheme dualism could not be boiled down to the claim that a conceptual scheme different from the interpreter's is largely true. In fact, the core sentences of an alien P-language have no truth-values when considered from the viewpoint of a competing P-language, not to mention that they are by no means 'largely true'. Thus, Davidson cannot derive translatability from truth since there are no shared truths between P-languages to begin with. Therefore, Davidson's argument from translatability against conceptual relativism does not apply to the presuppositional model.

'Wait a minute', a baffled critic might object, 'if your interpretation of scheme-differentiation makes sense, i.e., a truth-value gap occurs between two competing P-languages, then the result will be just like what the Quinean model concludes: truth-preserving translation between them is impossible. If so, Davidson's argument against untranslatability still has the teeth to tear off your very idea of conceptual scheme'.

The hypothetical critic is correct that my new criterion of scheme-differentiation, i.e., occurrence of a truth-value gap between two P-languages, does logically lead to the failure of truth-preserving intertranslatability between them. However, I would like

14 Similar to Davidson's notion of truth, the notion of truth-value status denotes a primitive irreducible concept. Just as Tarski's Convention T does not define the notion of truth but rather the truth predicate 'is true in L', what Convention P defines is not the notion of truth-value status, but rather the truth-value predicate 'is true-or-false in L'. 
to remind the critic that the Quinean thesis of untranslatability between alternative conceptual schemes itself is not inherently incoherent (although it is confusing and unproductive). It becomes incoherent only in conjunction with Davidson's interpretation of Quinean scheme-content dualism - that is, the dualism amounts to the claim that a conceptual scheme different from the interpreter's is largely true; but Davidson's truth conditional theory of translation does not allow the divorce between truth and translation. After removing the conjunct, the thesis of untranslatability is actually harmless (but also useless). Besides, it is not controversial at all to claim that intertranslatabilty is a sufficient condition for the identity of two conceptual schemes (if two languages are intertranslatable, then they embody the same conceptual scheme), nor its logical contrapositive, namely, that untranslatability is a necessary condition for alternative conceptual schemes (two languages embedded with two different conceptual schemes are untranslatable). But this is what the presuppositional model implies. What is at stake between the Quinean and the Davidsonian views is whether untranslatability is sufficient for scheme difference; or whether translatability is necessary for languagehood. The presuppositional model implies neither of these two controversial claims. This is because the failure of mutual translation between two languages does not logically lead to the occurrence of a truth-value gap between them.

\section{References}

Collingwood, R. G. 1940. An Essay on Metaphysics. Oxford: the Clarendon Press.

Davidson, D. 1984. Inquiries into Truth and Interpretation. New York: Oxford University Press.

Davidson, D. 2001. 'The Myth of the Subjectivity', in D. Davidson, Subjective, Intersubjective, Objective. Oxford: Clarendon Press, 39-52.

Feyerabend, P. 1978. Against Method. London: Verso.

Gaifman, H. 1975. 'Ontology and Conceptual Framework', part I, Erkenntnis 9, 329-53.

Gaifman, H. 1976. 'Ontology and Conceptual Framework', part II, Erkenntnis 10, 21-85.

Gaifman, H. 1984. 'Why Language?' in W. Balzer et al. (eds.), Reduction in Science. Boston: D. Reidel Publishing Company, 319-30.

Hacking, I. 1982. 'Language, Truth and Reason', in M. Hollis and S. Lukes (eds.), Rationality and Relativism. Cambridge: the MIT Press , 48-66.

Hacking, I. 1983. Representing and Intervening. New York: Cambridge University Press.

Hacking, I. 1992. " "Style” for Historians and Philosophers', Studies in the History and Philosophy of Science, 23 (1), 1-20.

Kuhn, T. 1991. 'The Road Since Structure', PSA 1990, vol. 2, the Philosophy of Science Association 1991, 3-13.

Kuhn, T. 1993. 'Afterword', in Horwich, P. (ed.), World Change: Thomas Kuhn and the Nature of Science. Cambridge: the MIT Press, 311-41.

Lynch, M. 1998. 'Three Models of Conceptual Schemes,' Inquiry 40, 407-26. 
Martin, J. 1975. 'A Many-Valued Semantics for Category Mistakes,' Synthese 31, 63-83.

Quine, W. V. 1980. 'Two Dogmas of Empiricism', in W. V. Quine, From a Logical Point of View, second edition. Cambridge: Harvard University Press, 20-46.

Quine, W. V. 1981. 'On the Very Idea of a Third Dogma', in W. V. Quine, Theories and Things. Cambridge: Harvard University Press, 39-42.

Rescher, N. 1980. 'Conceptual Schemes', in P. French, et al. (eds.), Studies in Epistemology. Minneapolis: University of Minnesota Press, 323-45.

Rorty, R. 1982. 'The World Well Lost', in R. Rorty, Consequences of Pragmatism. Minneapolis: University of Minnesota Press, 3-18.

Strawson, P. F. (1996). 'On Referring', in A. Martinich (ed.), The Philosophy of Language, third edition. New York: Oxford University Press, 215-30; originally published in Mind 59 (1950), 320-44.

Wang, X. 2009. 'On Davidson's Refutation of Conceptual Schemes and Conceptual Relativism', Pacific Philosophical Quarterly 90, 140-164.

Wang, X. 1999. 'Is the Notion of Semantic Presupposition Empty?' Diálogos 73, 61-91.

Wang. X. 1998. 'A Critique of the Translational Approach to Incommensurability,' Prima Philosophia, 11 (3), 293-306. 
Copyright of South African Journal of Philosophy is the property of Philosophical Society of Southern Africa (PSSA) and its content may not be copied or emailed to multiple sites or posted to a listserv without the copyright holder's express written permission. However, users may print, download, or email articles for individual use. 